\title{
Mediterranean diet, endothelial function and vascular inflammatory markers
}

\author{
Katherine Esposito ${ }^{1,2, *}$, Miryam Ciotola ${ }^{1}$ and Dario Giugliano ${ }^{1,2}$ \\ ${ }^{1}$ Division of Metabolic Diseases, University of Naples SUN, Naples, Italy: ${ }^{2}$ Center of Excellence for Cardiovascular \\ Diseases, University of Naples SUN, Naples, Italy
}

Submitted 20 April 2006: Accepted 10 November 2006

\begin{abstract}
Objectives: To discuss present knowledge about the relation between adipose tissue, inflammation and the Mediterranean-style diet.

Design: Review of the literature and personal perspectives.

Setting and results: Recent studies indicate that adipose tissue is an endocrine organ producing numerous proteins, collectively referred to as adipokines, with broad biological activity, which play an important autocrine role in obesity-associated complications. Adipose tissue in general and visceral fat in particular are thought to be key regulators of inflammation which is heavily involved in the onset and development of atherothrombotic disease. Moreover, chronic inflammation may also represent a triggering factor in the origin of the metabolic syndrome and type 2 diabetes mellitus. An increased release of proinflammatory adipokines from the visceral adipose tissue, associated with a reduced secretion of anti-inflammatory adipokines and cytokines, could determine a low-grade chronic inflammatory state which might play a role in the future development of the metabolic syndrome, diabetes and atherosclerosis through both insulin resistance and endothelial dysfunction. Interventions aimed at decreasing weight loss and improving adherence to a Mediterranean-style diet in people with obesity or metabolic syndrome decrease the inflammatory milieu and ameliorate both insulin resistance and endothelial dysfunction.

Conclusions: Appropriate dietary patterns, as those associated with the eating model of Mediterranean-type diets, represent therapeutic strategies to reduce inflammation and the associated metabolic and cardiovascular risk.
\end{abstract}

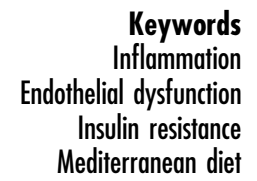

Keywords

dial dysfunction

Insulin resistance
During the past 10 years, the prevalence of obesity in the USA has increased from 23 to $31 \%$ of the country's 280 million people ${ }^{1}$. The latest National Health and Nutrition Examination Survey shows that approximately 127 million people are overweight, 60 million are obese and 10 million have life-threatening morbid obesity. Current trends indicate that obesity will increase to 39\% by 2010 and with it morbid obesity. An estimated 325000 deaths, and between 4.3 and $5.7 \%$ of direct health costs (approximately 39-52 billion dollars) are attributed to obesity annually. Results from the 1998 Behavioural Risk Factor Surveillance Survey indicate that roughly one-third of the USA adults were trying to lose weight at that time, and another third were trying to maintain weight ${ }^{2}$. Hence, the rate of obesity and the number of dieters are increasing in parallel. Obesity is not only an American phenomenon. The worldwide prevalence is increasing at such rapid pace that a WHO consultation on obesity designated obesity as the major unmet public health problem worldwide ${ }^{3}$.

\section{Obesity and inflammation}

Adipose tissue was for a long time regarded as a relatively passive site of energy store. This energy is accumulated in the form of triglycerides during the period of excess food consumption and mobilised when calorie intake is inadequate. Recent studies indicate that adipose tissue is an endocrine organ producing numerous proteins, collectively referred to as adipokines, with broad biological activity, which play an important autocrine role in physiology and are involved in obesity-associated complications ${ }^{4}$. A growing body of evidence implicates adipose tissue in general and visceral adiposity in particular as key regulators of inflammation. As adipose tissue secretes proinflammatory cytokines, it becomes linked at the molecular level to the dysregulation of a variety of underlying systems all of which are causally implicated in the development of atherosclerosis and metabolic outcomes.

Inflammation plays an important role in mediating all phases of atherogenesis, from the initial recruitment of 
circulating cells to the inner arterial layer to the weakening of the fibrous cap of the plaque, eventually leading to rupture. Inflammation is heavily involved in the onset and development of atherothrombotic disease, which is accompanied by the emergence of numerous inflammatory biomarkers, including cytokines as the interleukins, acute-phase proteins, adhesion molecules, interferons, chemokines, etc. Several studies have demonstrated an association between these biomarkers and current or future events of coronary artery disease ${ }^{5}$. A close relation is also present between the biomarkers and glucose metabolism abnormalities. For instance, chronic inflammation may also represent a triggering factor in the origin of the metabolic syndrome. Stimuli, such as overnutrition, physical inactivity and ageing would result in cytokine hypersecretion and eventually lead to insulin resistance and diabetes in genetically or metabolically predisposed individuals ${ }^{6}$.

\section{Proinflammatory cytokines}

Interleukin-6 (IL-6) is a pleiotropic-circulating cytokine with effects ranging from inflammation to host defence to tissue injury ${ }^{7}$. Adipose cells contribute to one-third of circulating IL-6 concentration, with visceral depots releasing more IL-6 when compared with sc depots. Plasma IL-6 levels positively correlate with human obesity and insulin resistance, and elevated levels of IL- 6 predict the development of type 2 diabetes $^{8}$ and future myocardial infarction?. The relative risk of future type 2 diabetes mellitus for women in the highest vs. lowest quintiles of these inflammatory markers was 7.5. Plasma IL-6 and insulin sensitivity relationships seem to occur in parallel to increases in plasma FFA. Finally, IL-6 may also decrease insulin sensitivity by reducing adiponectin secretion ${ }^{10}$. Large quantities of IL- 6 are found in human atherosclerotic plaques. Moreover, IL-6 impairs endothelium-dependent dilatation in human veins in vivo ${ }^{11}$.

So far, the potential mechanism proposed to explain the effect of tumour necrosis factor- $\alpha$ (TNF- $\alpha$ ) on obesityrelated insulin resistance includes increased release of FFA by adipocytes, reduction in adiponectin synthesis and impairment of insulin signalling ${ }^{12}$. Induction of insulin resistance by TNF- $\alpha$ is mediated through its ability to produce serine phosphorylation of insulin receptor substrate-1, decreasing the tyrosine kinase activity of the insulin receptor. Increased plasma levels of the soluble fraction of TNF receptor 2 have been found associated with insulin resistance in healthy volunteers, in lean nondiabetic offspring of type 2 diabetic subjects, and in young obese subjects $^{13}$. Moreover, infusion of exogenous TNF- $\alpha$ determines endothelial dysfunction in normal subjects linked to insulin resistance ${ }^{14}$. The synthesis of adipose tissue TNF- $\alpha$, as measured by reverse transcription polymerase chain reaction in abdominal subcutaneous adipose tissue samples, could induce the production of IL-6, CRP and other acute-phase reactants, thus contributing to the maintenance of chronic low-grade inflammation state involved in the progression of obesity.

Although C-reactive protein (PCR) is not an adipokine, its circulating concentrations are under the control of adipokines and its role in inflammation is well defined. Cross-sectional analyses have shown strong and independent associations of CRP levels with measures of body fat $^{15,16}$. High levels of CRP in obesity also predict later development of diabetes ${ }^{17}$. Rapidly evolving work now demonstrated that in addition to being a marker on innate immunity, CRP also has several direct effects at the level of vessel wall ${ }^{18}$. CRP is not only an inflammatory marker of atherosclerosis or coronary events, but also a mediator of the disease because it contributes to the substrate underlying lesion formation, plaque rupture and coronary thrombosis by promoting endothelial cell activation. Human recombinant CRP, at concentrations known to predict vascular disease, elicits a multitude of effects on endothelial biology favouring a proinflammatory and proatherosclerotic phenotype.

\section{Anti-inflammatory cytokines}

Adiponectin is an adipocyte-derived plasma protein with insulin sensitising, anti-inflammatory and anti-atherogenic properties. In human studies, plasma adiponectin levels are negatively correlated with obesity, anthropometric indices and insulin resistance, diabetic dyslipidaemia and cardiovascular disease ${ }^{5}$. Moreover, low plasma adiponectin levels are an independent risk factor for future development of type 2 diabetes $^{19}$. Therefore, hypoadiponectinaemia may be an important contributor to insulin resistance and the metabolic syndrome. Although the mechanisms by which adiponectin may ameliorate insulin resistance are not entirely clear, it decreases circulating FFA by increasing their oxidation by skeletal muscle ${ }^{20}$, with resultant decreased triglyceride content and hence improved insulin sensitivity. The reasons why adiponectin levels are low in obesity might be due to insulin resistance in the adipocyte, which attenuates or blunts insulinstimulated adiponectin secretion, or by increased suppression of its secretion by TNF- $\alpha$ and IL- $6^{12}$, whose levels are reported to be raised in obesity. Our findings of an inverse correlation between adiponectin increase and TNF- $\alpha$ decrease after weight loss in obese women ${ }^{21}$ seem to give some credit to the hypothesis that raised TNF- $\alpha$ circulating levels in obesity are causally linked to reduced adiponectin concentrations. In addition to its effects on fuel homeostasis, adiponectin displays anti-inflammatory properties.

IL-10 is a centrally operating cytokine secreted by activated monocytes/macrophages and lymphocytes, and possesses multifaceted anti-inflammatory and insulinsensitising properties by antagonising IL- 6 and TNF- $\alpha$. A low production capacity of IL-10 has been found to be associated with the metabolic syndrome and type 2 
diabetes ${ }^{22-24}$. Experimental studies using gene-targeted mice $^{25}$ provide direct evidence that IL-10 offers some protection against endothelial dysfunction during diabetes and that this effect is mediated by inhibition of increased in superoxide anion in blood vessels. A reduced IL-10 serum level represents a marker of plaque instability favouring the development of acute coronary syndromes, and also indicative of a poor prognosis even after the occurrence of an acute ischaemic event caused by plaque instability ${ }^{26}$.

\section{A working hypothesis}

On the basis of the available evidence described above, it could be hypothesised that an increased release of proinflammatory adipokines, mainly IL-6 and TNF- $\alpha$, from the visceral adipose tissue, associated with a reduced secretion of anti-inflammatory adipokines and cytokines, mainly adiponectin and IL-10, can determine a low-grade chronic inflammatory state. The proinflammatory milieu generated by the imbalance between pro- and antiinflammatory adipokines and cytokines might play a role in the future development of the metabolic syndrome, diabetes and atherosclerosis through both a reduced sensitivity of target tissues to insulin (insulin resistance) and a reduced anti-atherosclerotic function of the endothelium (endothelial dysfunction).

\section{Healthy lifestyle and inflammation}

The goal of obesity therapy is weight reduction, mainly through dieting and exercise. Even modest weight loss of $10 \%$ or less is associated with a substantial improvement in coexisting illnesses ${ }^{27}$. For example, in a randomised controlled study conducted in 120 obese women, we demonstrated that a multidisciplinary program aimed at reducing weight through lifestyle changes (diet and exercise) was associated with amelioration in circulating markers of vascular inflammation, including reduced levels of CRP and IL-6, and increased levels of adiponectin, associated with improved insulin sensitivity ${ }^{21}$. Unfortunately, our current urbanised, sedentary lifestyle and super-sized portions of food (characterised by our culturally oriented, chronic consumption of easily obtainable energy-dense liquids and foods) lead to fat storage, to which our hunter-gatherer species is genetically predisposed.

We explored possible mechanisms underlying a dietary intervention in patients at increased cardiac risk ${ }^{28}$. We randomised 180 patients (99 men and 81 women) with the metabolic syndrome to a Mediterranean-style diet (instructions about increasing daily consumption of whole grains, vegetables, fruits, nuts and olive oil) vs. a cardiac-prudent diet with fat intake less than 30\%. Physical activity increased equally in both the groups. After 2 years, body weight decreased more in the intervention group than in the control group, but even after controlling for weight loss, inflammatory markers, such as IL-6, IL-7, IL-18 and C-reactive protein, and insulin resistance declined more in the intervention group than in the control group, while endothelial function improved. Only 40 patients in the intervention group still had metabolic syndrome after 2 years when compared with 78 patients on the control diet; thus, the prevalence of the metabolic syndrome was reduced approximately by one half. These results suggest a plausible mechanism for the beneficial effects of a Mediterranean-style diet.

\section{Conclusions}

Obesity is a growing health problem. Excess weight and obesity increase morbidity and mortality associated with numerous complications, including type 2 diabetes mellitus, hyperlipidaemia, hypertension and atherosclero$\operatorname{sis}^{27}$. Adipose tissue is both a dynamic endocrine organ, as well as a highly active metabolic tissue. Fat produces and secretes adipokines, which are known to play an important role in the induction of insulin resistance and in the atherosclerotic process. Visceral adiposity is more strongly associated with insulin resistance than subcutaneous fat. However, the precise chain of events linking overnutrition, obesity, activation of the innate immune system and reduced insulin sensitivity in peripheral tissues remains incompletely understood. No specific dietary recommendations have been advocated by health agencies for treatment of insulin resistance or the metabolic syndrome. Given that the metabolic syndrome is an identifiable and potentially modifiable risk state for both type 2 diabetes and cardiovascular disease, adopting a dietary pattern based on increased consumption of fruit and vegetables, whole-grain cereal fibre, olive oil and nuts may reduce the potential risk of these diseases.

\section{References}

1 Flegal KM, Carroll MD, Ogden CL, Johnson CL. Prevalence and trends in obesity among US adults, 1999-2000. JAMA 2002; 288: 1723-7.

2 Serdula M, Mokdad AH, Williamson DF, Galuska DA, Mendlein JM, Heath GW. Prevalence of attempting weight loss and strategies for controlling weight. JAMA 1999; 282 1353-8.

3 WHO. Obesity: Preventing and Managing the Global Epidemics. Geneva: World Health Organization, 1998.

4 Trayhurn P, Beattie JH. Physiological role of adipose tissue: white adipose tissue as an endocrine and secretory organ. The Proceedings of the Nutrition Society 2001; 60: 329-39.

5 Berg AH, Scherer PE. Adipose tissue, inflammation, and cardiovascular disease. Circulation Research 2005; 96: 939-49.

6 Pickup JC. Inflammation and activated innate immunity in the pathogenesis of type 2 diabetes. Diabetes Care 2004; 27: 813-23.

7 Papanicolaou DA, Wilder RL, Manolagas SC, Chrousos GP. The pathophysiologic roles of interleukin-6 in human disease. Annals of Internal Medicine 1998; 128: 127-37. 
8 Pradhan AD, Manson JE, Rifai N, Buring JE, Ridker PM. C-reactive protein, interleukin 6, and risk of developing type 2 diabetes mellitus. JAMA 2001; 286: 327-34.

9 Ridker PM, Rifai N, Stampfer MJ, Hennekens CH. Plasma concentration of interleukin-6 and the risk of future myocardial infarction among apparently healthy men. Circulation 2000; 101: 1767-72.

10 Fasshauer M, Kralisch S, Klier M, Lossner U, Bluher M, Klein J, et al. Adiponectin gene expression and secretion is inhibited by interleukin-6 in 3T3-L1 adipocytes. Biochemical and Biophysical Research Communications 2003; 301: 1045-50.

11 Bhagat K, Valance P. Inflammatory cytokines impair endothelium dependent dilatation in human veins in vivo. Circulation 1997; 96: 3042-7.

12 Bruun JM, Lihn AS, Verdich C, Pedersen SB, Toubro S, Astrup A, et al. Regulation of adiponectin by adipose tissue-derived cytokines: in vivo and in vitro investigations in humans. American Journal of Physiology. Endocrinology and Metabolism 2003; 285: E527-33.

13 Dzienis-Straczkowska S, Straczkowski M, Szelachowska M, Stepien A, Kowalska I, Kinalska I. Soluble TNF $\alpha$ receptors in young obese subjects with normal and impaired glucose tolerance. Diabetes Care 2003; 26: $875-80$.

14 Rask-Madsen C, Domínguez H, Ihleman N, Hermann T, Kober L, Torp-Pedersen C. Tumor necrosis factor- $\alpha$ inhibits insulin's stimulating effect on glucose uptake and endothelium-dependent vasodilation in humans. Circulation 2003; 108: 1815-21.

15 Festa A, D'Agostino R Jr, Howard G, Mykkanen L, Tracy RP, Haffner SM. Chronic subclinical inflammation as part of the insulin resistance syndrome. The Insulin Resistance Atherosclerosis Study (IRAS). Circulation 2000; 102: 42-7.

16 Ziccardi P, Nappo F, Giugliano G, Esposito K, Marfella R, Cioffi $\mathrm{M}$, et al. Reduction of inflammatory cytokine concentrations and improvement of endothelial functions in obese women after weight loss over one year. Circulation 2002; 105: 804-9.

17 Han TS, Sattar N, Williams K, Gonzales-Villalpando C, Lean ME, Haffner SM. Prospective study of C-reactive protein in relation to the development of diabetes and metabolic syndrome in the Mexico City Diabetes Study. Diabetes Care 2002; 25: 2016-21.
18 Pasceri V, Willerson JT, Yeh ET. Direct proinflammatory effect of C-reactive protein on human endothelial cells. Circulation 2000; 102: 2165-8.

19 Lindsay RS, Funahashi T, Hanson RL, Matsuzawa Y, Tanaka $\mathrm{S}$, Tataranni PA, et al. Adiponectin and development of type 2 diabetes in the Pima Indian population. Lancet 2002; 360: 57-8.

20 Yamauchi T, Kamon J, Waki H, Terauchi Y, Kubota N, Hara $\mathrm{K}$, et al. The fat-derived hormone adiponectin reverses insulin resistance associated with both lipoatrophy and obesity. Nature Medicine 2001; 7: 941-6.

21 Esposito K, Pontillo A, Di Palo C, Giugliano G, Masella M, Marfella R, et al. Effect of weight loss and lifestyle changes on vascular inflammatory markers in obese women: a randomized trial. JAMA 2003; 289: 1799-804.

22 van Exel E, Gussekloo J, de Craen AJ, Frolich M, BootsmaVan Der Wiel A, Westendorp RG. Leiden 85 Plus Study. Low production capacity of interleukin-10 associates with the metabolic syndrome and type 2 diabetes: the Leiden 85-Plus Study. Diabetes 2002; 51: 1088-92.

23 Esposito K, Pontillo A, Giugliano F, Giugliano G, Marfella R, Nicoletti G, et al. Association of low interleukin-10 levels with the metabolic syndrome in obese women. The Journal of Clinical Endocrinology and Metabolism 2003; 88: 1055-8.

24 Esposito K, Giugliano D, Nappo F, Marfella R. Campanian Postprandial Hyperglycemia Study Group. Regression of carotid atherosclerosis by control of postprandial hyperglycemia in type 2 diabetes mellitus. Circulation 2004; 110: $214-9$.

25 Gunnett CA, Heistad DD, Faraci FM. Interleukin-10 protects nitric oxide-dependent relaxation during diabetes: role of superoxide. Diabetes 2002; 51: 1931-7.

26 CAPTURE Study Investigators, Heeschen C, Dimmeler S, Hamm CW, Fichtlscherer S, Boersma E, Simmons ML, et al. Serum level of the antiinflammatory cytokine interleukin-10 is an important prognostic determinant in patients with acute coronary syndromes. Circulation 2003; 107: 2109-14.

27 Kopelman PG. Obesity as a medical problem. Nature 2000; 404: $635-43$.

28 Esposito K, Marfella R, Ciotola M, Di Palo C, Giugliano F, Giugliano $G$, et al. Effect of a Mediterranean-style diet on endothelial dysfunction and markers of vascular inflammation in the metabolic syndrome: a randomized trial. JAMA 2004; 292: 1440-6. 